\section{On the Variability of Lung Function Measurements in the Horse - A Preliminary Report}

\author{
E. Deegen and H.J. Klein
}

Klinik für Pferde

Tierärztliche Hochschule Hannover

During the measurement of lung function parameters, the measuring process influences test parameters in patients not adapted to the equipment. These effects can change the lung function parameters as can other influences, e.g., circadian rhythm (Stadler et al., 1985).

In the literature, attention has been drawn to the fact that the use of esophageal balloon probes requires an adaptation time on the esophagus to the inserted probe. Before starting the measuring process, most authors observe an adaptation time of 5 to 10 minutes (Klein, 1984; Sass, 1985).

It was our aim to evaluate the changes in lung function during this adaptation period. Our studies have shown that in most horses suffering from chronic bronchitis the values for dynamic compliance $\left(\mathrm{C}_{\text {dyn }}\right)$, total pulmonary resistance $\left(R_{t p}\right)$ and work of the respiratory system $\left(W_{r s}\right)$ got worse after adaptation to the instruments. This was observed particularly in patients, who, after initial nervousness, calmed down during the measuring procedure. Other patients remained nervous during the whole measuring procedure. In those cases, the lung function parameters usually did not show any typical changes (Fig. 1).

We state the following hypothesis for those changes in lung function: stress and nervousness at the beginning of lung function testing induces the release of endocrine catecholamines, which, particularly in horses with bronchospasm, lead to short-term bronchodilation and thus improvement in lung function. After metabolism of the catecholamines, lung function seems to return to lower values for $C_{d y n}$ and higher values for $R_{t p}$ and $W_{r s}$.

Therefore, we studied the adrenaline effect in two horses with chronic obstructive pulmonary disease (COPD). We selected two of our own horses suffering from severe COPD which had been tested for lung function many times. The horses were completely adapted to the equipment. Within the first 10 minutes after starting the measurements, neither patient showed any changes in lung function parameters. In this study, as well as in our previous investigations, we used $\mathrm{C}_{\mathrm{dyn}}$ as the most sensitive test parameter. As already described by Stadler et al. (1985), $\mathrm{C}_{\mathrm{dyn}}$ was determined by means of the loop diagonal constructed from the pressure-volume loops.

The weights of the horse were 550 and $580 \mathrm{~kg}$. Adrenaline was given intravenously starting at a dose of $0.0625 \mathrm{mg}$ per horse. The dose was doubled every 5 minutes until a dose of $1 \mathrm{mg}$ was reached. Then the amount of adrenaline was not increased any more, because after administration of a

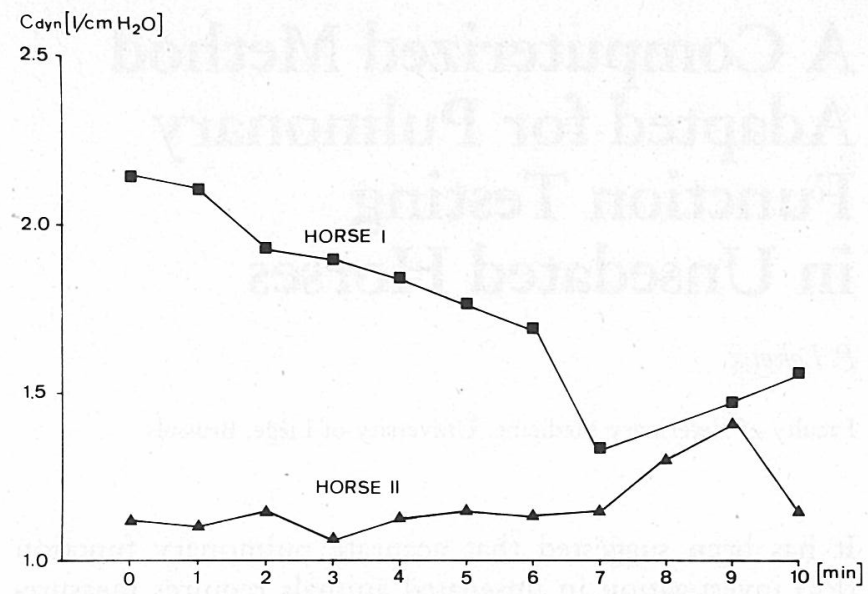

Fig. 1: Changes of the dynamic compliance $\left(\mathrm{C}_{\mathrm{dyn}}\right)$ in horses after application of the measuring equipment. Horse I represents a group of horses, which calmed down during the measuring procedure after initial nervousness. In some other horses, like horse II, lung function parameters do not show any typical changes.

dose of $1 \mathrm{mg}$ both animals showed marked signs of nervousness. At a dose of $0.25 \mathrm{mg}$ the animals started sweating, and at a dose of $0.5 \mathrm{mg}$ adrenaline muscle-trembling was observed. The first three doses of adrenaline caused a spontaneous short-term increase in heart rate to about 60 beats/min. The last two doses of adrenaline, 0.5 and $1 \mathrm{mg}$, produced a somewhat delayed rise up to about 70 beats/ $\min$.

The two test protocols demonstrate that a dose-dependent improvement in $\mathrm{C}_{\mathrm{dyn}}$ occurred up to a dose of $0.5 \mathrm{mg}$ adren-

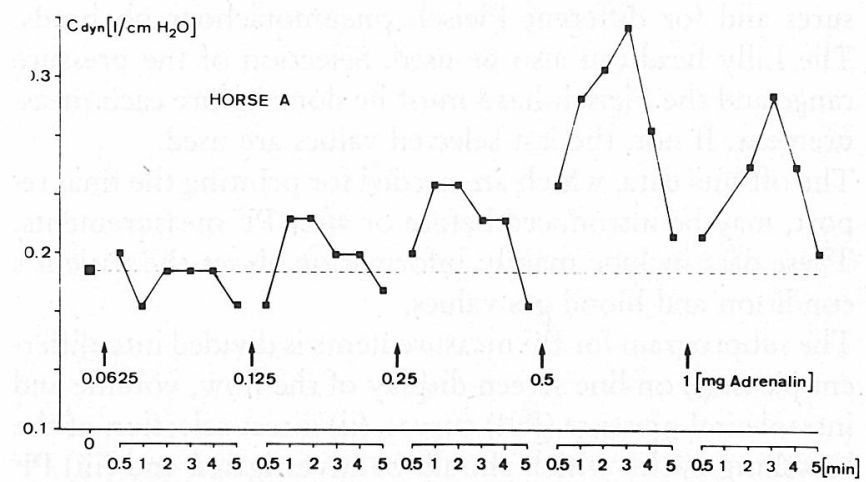

Fig. 2 and 3: Test protocols of the 2 horses (" $A$ " and " $B$ ") following increasing doses of adrenaline. Dashed line $=$ base line value of $C_{\text {dyn }}$.

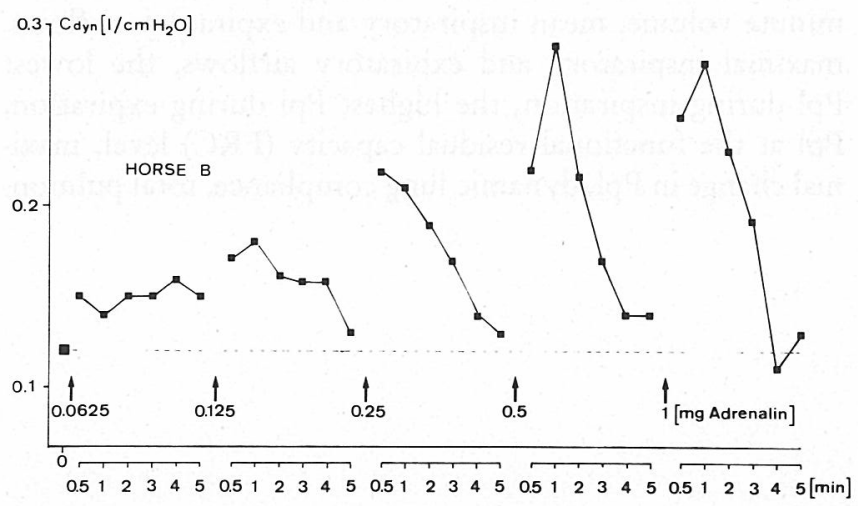


aline (Figs. 2 and 3). After application of $1 \mathrm{mg}$ adrenaline, no further improvement could be observed in either patient. The duration of the adrenaline effect was about 4 minutes. Five minutes after injection, the effects of all doses of adrenaline had dissipated.

The test results show that in horses suffering from COPD, adrenaline can cause a marked short-term improvement in lung function. Under stress, horses are able to spontaneously release large amounts of catecholamines.

These beta-2-agonist catecholamines improve lung function, especially when the subjects are visibly nervous during the measurements. Additionally this investigation shows that lung function may have already been influenced before sweating or nervousness have been seen in the horse.

Finally, the study gave some information on the duration of adrenaline-induced bronchodilation in horses.

\section{References}

Klein, H.J. (1984): Der Histamininhalationsprovokationstest zur Bestimmung der unspezifischen Reagibilität der Atemwege beim Pferd. Hannover, Tierärztl. Hochsch., Diss.

Sass, O. (1985): Lungenfunktionsanalysen vor und nach massiver Infusionsbehandlung bei Pferden mit chronischen Bronchialerkrankungen. Hannover, Tierärztl. Hochsch., Diss.

Stadler, P., Deegen, E., and Reinhard, H.J. (1985): Circadian rhythm of lung function parameters in horses with chronic airway diseases. Meeting "Lung function and respiratory diseases in horses", Hannover, June 1985.

Prof. Dr. E. Deegen

Klinik für Pferde

Tierärztliche Hocbschule Hannover

Bischofsholer Damm 15

D.3000 Hannover 1, Germany 